\title{
Burden and Suffering in Chronic Venous Disease
}

Andrew N. Nicolaides $\cdot$ Nicos Labropoulos

Received: January 17, 2019 / Published online: February 13, 2019

(C) The Author(s) 2019

\begin{abstract}
Chronic venous disease (CVD) is widespread, underdiagnosed, and can progress to chronic venous insufficiency and venous ulcers, which can require extensive treatment and hospitalization. These conditions negatively impact patient quality of life and place substantial burdens on healthcare resources. The two main risk factors for CVD are age and obesity. Thus, with the growing prevalence of obesity and the increasing longevity of the population, the burden of CVD is expected to increase dramatically in the coming decades. Appropriate lifestyle changes and care, which may include treatment with venoactive drugs, can slow disease progression, improve quality of life, and are likely to reduce healthcare costs. Physicians should be aware of this growing problem and of the effective treatments available for CVD. We recommend the accompanying short summaries from a symposium held at the recent
\end{abstract}

Enhanced Digital Features To view enhanced digital features for this article go to https://doi.org/10.6084/ m9.figshare.7599158.

A. N. Nicolaides $(\square)$

Vascular Screening and Diagnostic Centre, Nicosia, Cyprus

e-mail: anicolaides1@gmail.com

N. Labropoulos

Department of Surgery, Stony Brook University

Medical Center, Stony Brook, NY, USA
European Venous Forum as a means for our colleagues to learn more about the burden and suffering associated with CVD.

Funding: Servier.

Keywords: Cardiology; Chronic venous disease; Chronic venous insufficiency; Venoactive drugs; Venoactive drug therapy

Poor venous circulation in the legs is the source of many common health complaints worldwide. The early symptoms of chronic venous disease (CVD), including leg pain, swelling, and feelings of leg heaviness, can be continuous or can appear or increase in severity toward the end of the day. Patients often seek initial treatment for symptoms that present with or without varicose veins or for cosmetic treatment of asymptomatic varicose veins. As the disease worsens or spreads to other leg areas, patients may develop chronic edema, skin hardening and darkening, and venous ulcers. Increasing age and excess body mass are the main risk factors for CVD. Other risk factors are related to genetics, female gender, and sedentary living habits or occupations. Without appropriate treatment and/or elimination of lifestyle or risk factors, disease progression and diminished quality of life (QoL) are inevitable.

At the 19th annual meeting of the European Venous Forum (EVF; June 28-30, 2018, Athens, 
Greece), a symposium entitled "Burden and Suffering in Chronic Venous Disease" sponsored by Servier (France) was held to discuss the burden, symptoms, QoL, progression, and treatment of patients with CVD. The symposium panel included three experts in CVD research and treatment: Professor Alun H. Davies from Imperial College London, UK; Professor Nicos Labropoulos from Stony Brook University Medical Center, Stony Brook, NY; and Professor Jorge H. Ulloa from the Vascular Foundation, Bogota, Colombia.

The global burden of CVD care and treatment is already enormous, consuming approximately $2 \%$ of national healthcare budgets, and will continue to increase as the population of older adults grows and the prevalence of obesity rises [1-4]. In the UK alone, the proportion of adults over the age of 85 is projected to double to $4.5 \%$ in the next 25 years [5]. Evidence for the increasing healthcare burden can also be seen in varicose vein incidence rates of $4-5 \%$ over 2 years [6], disease progression rates of 4\% [7-9], and in statistics for varicose vein procedures, which have been projected to increase by $60 \%$ between 2013 and 2021 [10]. Even in patients who have had their varicose veins removed, many patients experience clinical recurrence and disease progression in previously unaffected veins $[11,12]$. To make matters worse, CVD patients are at risk of receiving inappropriate treatment because the general practitioners responsible for treating them may not recognize venous disorders or their seriousness, or may not refer them to vein specialists [3]. The key message here is that the numbers of CVD patients stand to increase substantially in the coming years and decades.

To prevent disease progression and limit the coming healthcare burden as much as possible, patients need early and appropriate treatment and lifestyle counseling, if necessary, to reduce excess body mass and attain healthy exercise habits. For this to happen consistently, physicians need to recognize the symptoms and signs of early stage CVD, understand the consequences of inadequate treatment and disease progression, and either become aware of the most effective treatment strategies available or refer their patients to specialists who can manage the disease effectively.

While only prevention strategies can stem the growing tide of new CVD patients, appropriate management of patients with early stage venous disease could help limit the number of patients who progress to severe CVD and develop venous ulcers, the care of which represents a major proportion of the CVD healthcare burden. CVD progression and the signs and symptoms of CVD are all due to a vicious cycle of venous hypertension and inflammation. Chronic hypertension leads to vein wall distension and endothelium disturbances that precipitate the adherence and activation of leukocytes that initiate an inflammatory cascade. Chronic inflammation, in turn, leads to additional changes at the vein wall and at venous valves, which contribute to worsening venous hypertension, blood pooling, and varicose vein development. Inflammation also leads to pathological changes in the microcirculation, where excess permeability and red blood cell infiltration lead to edema, tissue damage, and venous ulcers.

All CVD patients are prone to disease progression, though the rate and extent of progression depend on patient risk factors. In the Bonn Vein Study, 32\% of patients with early stage disease (C2) and reflux in the greater saphenous vein progressed to a higher CEAP class within 6 years [13]. In the Edinburgh Vein Study, $57.8 \%$ of CVD patients had progressed to a higher CEAP class or developed new varicose veins after a mean follow-up period of 13.4 years, with an annual progression rate of $5 \%$ [7]. Clearly, disease progression will contribute to the growing burdens of CVD on healthcare expenditures and on patient QoL. More studies are needed to identify the risk factors for progression to venous ulcers and to define the patients who can benefit from early vein interventions.

Conservative interventional treatments that reduce venous hypertension and inhibit the inflammatory cascade can alleviate symptoms and improve QoL [14]. In general, weight control and regular exercise should be recommended for all CVD patients to slow disease progression. Compression therapy, where 
applicable, is often the first treatment option for patients with varicose veins and can improve symptoms. However, compression therapy has not been shown to slow progression or prevent the recurrence of varicose veins [15]. Further, patient compliance can be low because the stockings can be uncomfortable. Thus, compression therapy alone is likely to be insufficient for most patients.

On the other hand, therapies with venoactive drugs provide promising and likely cost-effective alternative treatment options, because a number of these drugs have been demonstrated to improve the signs and symptoms of CVD and to slow progression [14]. Of these, micronized purified flavonoid fraction (MPFF/Daflon ${ }^{\circledR}$ ) is perhaps the most well-studied example. In animal models of venous hypertension, MPFF administration prevents leukocyte adhesion and rolling in hypertonic veins, and prevents pathological changes in the microvasculature [16]. In CVD patients, MPFF treatment steadily and significantly reduced leg pain over a period of 8 weeks [17], and improved leg discomfort and heaviness [18]. Importantly, MPFF also significantly improved QoL as assessed by the validated CIVIQ-20 test [18]. For these and other positive results obtained from randomized clinical trials, MPFF received grade A ratings in the latest EVF, International Union of Angiology (IUA), and International Union of Phlebology (UIP) guidelines for efficacy in improving signs, symptoms, and QoL due to CVD [14]. In addition, MPFF and other venoactive drugs should be very cost-effective because for most signs and symptoms less than five patients need to be treated to observe significant improvement in at least one patient [14].

From the study results presented at this symposium, it is clear that CVD will continue to impose huge and growing burdens on the people it afflicts and on healthcare systems worldwide. This problem needs urgent attention by the medical community to prevent unnecessary suffering and healthcare expenditure. Effective treatments are available and likely to be very cost-effective, but more studies are needed to confirm their cost-effectiveness and to provide the rationale for patient reimbursement. We urge our colleagues to consider the accompanying summaries as starting points to improve their understanding of the scope of CVD and the treatment strategies available.

\section{ACKNOWLEDGEMENTS}

Funding. This supplement has been sponsored by Servier. Article processing charges and the open access fee for this editorial were funded by Servier. All authors had full access to all of the data for this study and take complete responsibility for the integrity of the data and accuracy of the data analysis.

Medical Writing. Medical writing services were provided by Dr Kurt Liittschwager (4Clinics, France) and funded by Servier.

Authorship. The authors meet the International Committee of Medical Journal Editors (ICMJE) criteria for authorship for this article, take responsibility for the integrity of the work as a whole, and have approved this version for publication.

Prior Presentation. This article and all of the articles in this supplement are based on the International satellite symposium at European Venous Forum, June 29th, Athens.

Disclosures. The symposium was funded by an educational grant from Servier. Andrew N. Nicolaides received honoraria from Servier for chairing the symposium and has received honoraria in the past for serving on the Servier speaker bureau. Nicos Labropoulos received honoraria from Servier for chairing the symposium and has received honoraria in the past for serving on the Servier speaker bureau. Nicos Labropoulos also received an honorarium from Servier for the symposium lecture.

Compliance with Ethics Guidelines. This article is based on previously conducted studies and does not contain any studies with human participants or animals performed by any of the authors. 
Open Access. This article is distributed under the terms of the Creative Commons Attribution-NonCommercial 4.0 International License (http://creativecommons.org/licenses/ by-nc/4.0/), which permits any noncommercial use, distribution, and reproduction in any medium, provided you give appropriate credit to the original author(s) and the source, provide a link to the Creative Commons license, and indicate if changes were made.

\section{REFERENCES}

1. Evans CJ, Fowkes FG, Ruckley CV, Lee AJ. Prevalence of varicose veins and chronic venous insufficiency in men and women in the general population: Edinburgh Vein Study. J Epidemiol Commun Health. 1999;53(3):149-53 (Epub 1999/07/09).

2. Laing W. Chronic venous diseases of the leg. London: Office of Health Economics; 1992.

3. Rabe E, Guex JJ, Puskas A, Scuderi A, Fernandez Quesada F. Epidemiology of chronic venous disorders in geographically diverse populations: results from the Vein Consult Program. Int Angiol. 2012;31(2):105-15 (Epub 2012/04/03).

4. Rice JB, Desai U, Cummings AK, Birnbaum HG, Skornicki M, Parsons N. Burden of venous leg ulcers in the United States. J Med Econ. 2014;17(5):347-56 (Epub 2014/03/15).

5. Office for National Statistics. National Population Projections: 2016-based statistical bulletin. In: Statistics OfN, editor. London: Office for National Statistics; 2016.

6. Brand FN, Dannenberg AL, Abbott RD, Kannel WB. The epidemiology of varicose veins: the Framingham Study. Am J Prev Med. 1988;4(2):96-101 (Epub 1988/03/01).

7. Lee AJ, Robertson LA, Boghossian SM, Allan PL, Ruckley CV, Fowkes FG, et al. Progression of varicose veins and chronic venous insufficiency in the general population in the Edinburgh Vein Study. J Vasc Surg Venous Lymphat Disord. 2015;3(1): 18-26 (Epub 2015/01/01).

8. Pannier F, Rabe E. The relevance of the natural history of varicose veins and refunded care. Phlebology. 2012;27(Suppl 1):23-6 (Epub 2012/02/15).
9. Pannier F, Rabe E. Progression in venous pathology. Phlebology. 2015;30(1 Suppl):95-7 (Epub 2015/03/ 03).

10. Onida S, Davies AH. Predicted burden of venous disease. Phlebology. 2016;31(1 Suppl):74-9 (Epub 2016/02/27).

11. Bush RG, Bush P, Flanagan J, et al. Factors associated with recurrence of varicose veins after thermal ablation: results of the recurrent veins after thermal ablation study. ScientificWorldJournal. 2014;2014: 505843 (Epub 2014/03/05).

12. O'Donnell TF, Balk EM, Dermody M, Tangney E, Iafrati MD. Recurrence of varicose veins after endovenous ablation of the great saphenous vein in randomized trials. J Vasc Surg Venous Lymphat Disord. 2016;4(1):97-105 (Epub 2016/03/08).

13. Rabe E, Pannier F, Ko A, Berboth G, Hoffmann B, Hertel $\mathrm{S}$. Incidence of varicose veins, chronic venous insufficiency, and progression of the disease in the Bonn Vein Study II. J Vasc Surg. 2010;51(3):791.

14. Nicolaides A, Kakkos S, Baekgaard N, et al. Management of chronic venous disorders of the lower limbs. Guidelines according to scientific evidence. Part I. Int Angiol. 2018;37(3):181-254 (Epub 2018/06/07).

15. Palfreyman SJ, Michaels JA. A systematic review of compression hosiery for uncomplicated varicose veins. Phlebology. 2009;24(Suppl 1):13-33 (Epub 2009/05/16).

16. das Graças C de Souza M, Cyrino FZ, de Carvalho JJ, Blanc-Guillemaud V, Bouskela E. Protective effects of micronized purified flavonoid fraction (MPFF) on a novel experimental model of chronic venous hypertension. Eur J Vasc Endovasc Surg. 2018;55(5):694-702 (Epub 2018/03/29).

17. Kirienko A, Radak D. Clinical acceptability study of once-daily versus twice-daily micronized purified flavonoid fraction in patients with symptomatic chronic venous disease: a randomized controlled trial. Int Angiol. 2016;35(4):399-405 (Epub 2015/11/19).

18. Carpentier P, van Bellen B, Karetova D, et al. Clinical efficacy and safety of a new 1000-mg suspension versus twice-daily 500-mg tablets of MPFF in patients with symptomatic chronic venous disorders: a randomized controlled trial. Int Angiol. 2017;36(5):402-9 (Epub 2017/02/17). 